\title{
Antimicrobial and Hemolytic Activity of Cysteine-Deleted Tachyplesin (CDT) Analogues in the Pursuit of Therapeutic Selectivity
}

\author{
Deborah Heyl, Yeji Park, Jennifer Garvey, Rebecca Newman, \\ and Yllka Vladaj \\ Department of Chemistry, Eastern Michigan University, Ypsilanti, MI, 48197, USA
}

\section{Introduction}

Because of the increasing resistance of bacteria to antibiotics, antimicrobial peptides (AMPs) may provide an alternative to traditional therapy. Tachyplesin is an AMP found in horseshoe crabs that can permeabilize the cell membrane [1]. Its positively charged residues are attracted to the negatively charged bacterial membrane. The hydrophobic amino acids then can insert into the nonpolar region of the membrane bilayer, forming pores or pulling the membrane apart. Human cell membranes are more zwitterionic, so the peptide does not affect them to the same extent. However, better selectivity for bacterial over mammalian cells is clearly desirable. Due to the mechanism by which AMPs act, bacteria are less able to develop resistance. Starting with a linear tachyplesin analogue that had the cysteine residues deleted and retained activity (CDT) [2], this study examines variations in end capping, sequence reversal, and the inclusion of D-amino acids.

\section{Results and Discussion}

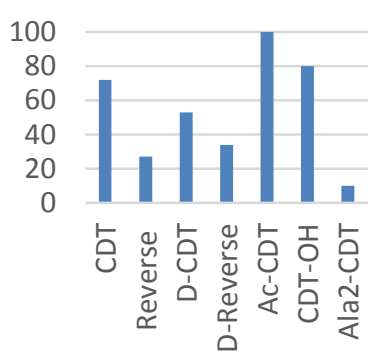

Fig. 1. Percent liposome dye leakage caused by $20 \mu \mathrm{M}$ peptide.
All analogues of CDT were synthesized by solid phase peptide synthesis using an Fmoc protection strategy on a PS3 Automated Peptide Synthesizer by Protein Technologies. They were then cleaved from the resin by trifluoroacetic acid, purified by RP-HPLC, and tested for effects on model membranes as well as for antimicrobial and hemolytic activity. Sequences can be found in Table 1. To test for the ability to disrupt liposomes, carboxyfluorescein encapsulated vesicles were made using a 3:1 mixture of POPC:POPG (palmitoyloleylphosphocholine and glycerol, respectively); dye leakage was measured in the presence of $20 \mu \mathrm{M}$ peptide and compared to that caused by a Triton-X detergent control (Figure 1). Antimicrobial activity was assessed by determining the minimum inhibitory concentration of peptide for gram positive $S$. aureus and gram negative E. coli strains (Table 1). Serial dilutions of the peptides were added to wells containing specific concentrations of bacteria. The cultures were allowed to grow overnight and turbidity readings were taken to measure bacterial growth. Damage to eukaryotic cells was evaluated spectrophotometrically by measuring hemolysis of sheep red blood cells compared to Triton-X treated controls (Table 1). Relative selectivity for bacterial over mammalian cells is represented in Figure 2, where the selectivity index was calculated by dividing the maximum concentration of peptide which caused less than $1 \%$ red blood cell hemolysis (where a high value is advantageous) by the MIC (where a low value indicates greater effectiveness).

Results for the first set of analogues show that reversing the amino acid sequence had virtually no effect on MIC values (CDT vs. Reverse CDT or All D-CDT vs. All D- Reverse CDT). However, the reverse sequences were less selective for bacteria, making them less promising as therapeutics.

Altering the stereochemistry, on the other hand, improved antimicrobial activity and selectivity. All-D Reverse CDT showed more selectivity than Reverse CDT, and All-D CDT exhibited a significant increase in selectivity over CDT. Changing the stereochemistry of amino acids did not improve membrane permeabilization (as expected, since membrane interaction does not involve specific binding), yet led to greater antibacterial activity in All D-CDT. This suggests that this D-amino acid containing peptide may have a secondary mechanism that targets bacterial cells. 
Table 1. Sequences of the antimicrobial peptides, effective minimum concentrations to inhibit bacterial growth (MIC), and concentration producing less than 1\% hemolysis.

\begin{tabular}{|c|c|c|c|c|}
\hline \multirow[b]{2}{*}{ Analog } & \multirow[b]{2}{*}{ Sequence } & \multicolumn{2}{|c|}{$M I C(\mu M)$} & \multirow{2}{*}{$\begin{array}{c}<1 \% \\
\text { Hemolysis } \\
(\mu M)\end{array}$} \\
\hline & & $\begin{array}{l}\text { E. coli } \\
\text { C25 }\end{array}$ & $\begin{array}{c}\text { S. aureus } \\
\text { ATCC } 25923\end{array}$ & \\
\hline CDT & KWFRVYRGIYRRR-NH ${ }_{2}$ & 7.1 & 14 & 67 \\
\hline Reverse CDT & RRRYIGRYVRFWK-NH ${ }_{2}$ & 8.4 & 16.8 & 8.4 \\
\hline All-D CDT & kwfrvyrgiyrrr- $\mathrm{NH}_{2}$ & 2.1 & 8.4 & 540 \\
\hline All-D Reverse CDT & rrryigryvrfwk- $\mathrm{NH}_{2}$ & 8.4 & 16.8 & 67 \\
\hline Ac-CDT & Ac-KWFRVYRGIYRRR-NH ${ }_{2}$ & 16.5 & 4.11 & 8.2 \\
\hline CDT-OH & KWFRVYRGIYRRR-OH & 33.7 & 67.3 & 17 \\
\hline $\mathrm{Ala}^{2}-\mathrm{CDT}$ & KAFRVYRGIYRRR-NH ${ }_{2}$ & 71.8 & 144 & 72 \\
\hline
\end{tabular}

Capping the $N$-terminus (Ac-CDT) or removal of the natural $C$-terminal "capping" carboxamide (CDT-OH) led to decreased selectivity for bacterial cells. Both peptides showed greater membrane disrupting ability in vitro, and more mammalian cell membrane peremabilization (hemolysis). Although acetylated CDT (Ac-CDT) showed slightly increased activity against $S$. aureus (the only analogue to show selectivity for the gram positive over the gram negative strain), these analogues, which both reduce overall positive charge, generally decreased both antimicrobial activity and selectivity, especially the CDT-OH with its $C$-terminal negative charge.

Replacement of Trp at the $2^{\text {nd }}$ position in CDT (Ala ${ }^{2}-\mathrm{CDT}$ ) reduced overall antimicrobial action. $\mathrm{Ala}^{2}$-CDT exibited the lowest antibacterial activity of all CDT analogues here (10-fold reduction relative to CDT), and also displayed very little hemolytic activity. The low dye leakage from vesicles also indicates that a lack of tryptophan at this position significantly decreases membrane permeabilization. The observation is consistent with the published literature, which states that Trp in

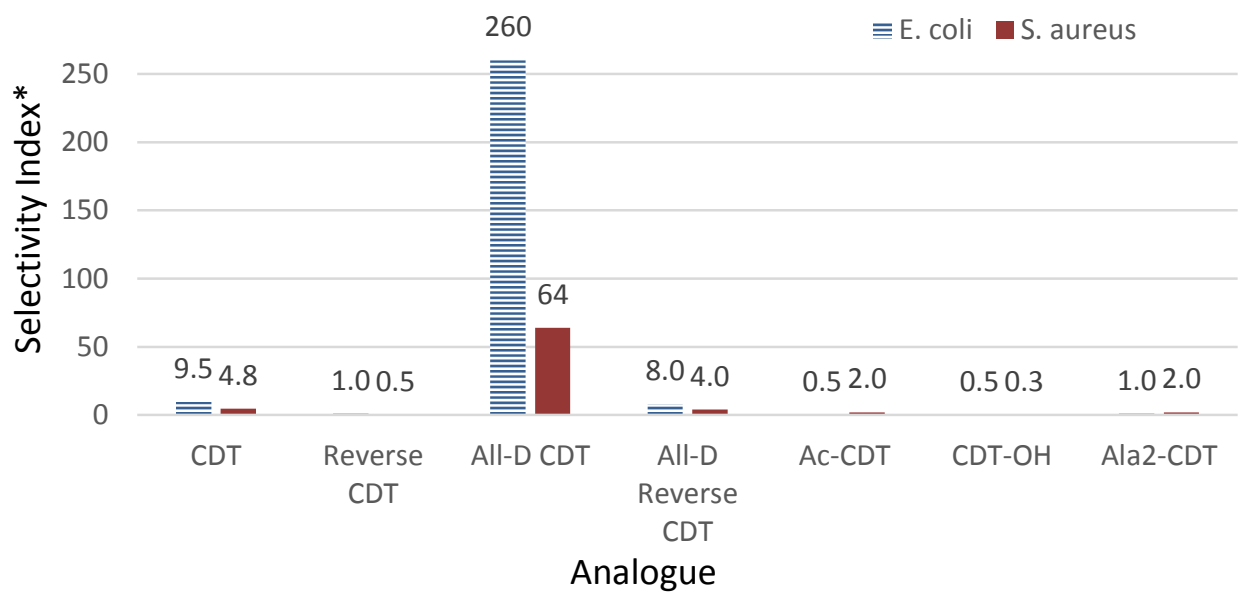

Fig. 2. Relative selectivity of each analogue for bacterial over mammalian cells; for example, at 64 times the concentration required to kill S. aureus, All-D CDT causes $<1 \%$ RBC hemolysis.

*S.I. is calculated by dividing the concentration of peptide for which there is $<1 \%$ hemolysis by the MIC. This gives an indication of selectivity for bacterial over mammalian cells. 
antimicrobial peptides, including CDT, enhances membrane permeabilization and strengthens antimicrobial activity [3].

All D-CDT exhibited the best bactericidal activity and highest selectivity index of all CDT analogues developed thus far [4]. While the MIC is improved only about 1.5 to 3 -fold against $S$. aureus and E. coli, respectively, its selectivity was significantly better than that of CDT itself since it caused virtually no damage to red blood cells, even at much higher concentrations than those required to kill the bacteria. D-amino acids also afford resistance against enzymatic breakdown and less immunogenicity. This analogue therefore shows broad spectrum activity with the potential to be developed into a potent nontoxic therapeutic drug.

\section{Acknowledgments}

Sincere thanks to Dr. Jim Vandenbosch for help with the MIC assays. This work was supported by the Eastern Michigan University Graduate School, and the Provost's Research Support and Chemistry Department Seller's Funds.

\section{References}

1. Nakamura, T., Furunaka, H., Miyata, T., Tokunaga, F., Muta, T., Iwanaga, S., Niwa, M., Takao, T., Shimonishi, Y. J. Biol. Chem. 263, 16709-6713 (1988), http://www.ncbi.nlm.nih.gov/pubmed/3141410

2. Ramamoorthy, A., Thennarasu, S., Tan, A., Gottipati, K., Sreekumar, S., Heyl, D.L., An, F., Shelburne, C.E. Biochemistry 45, 6529-6540 (2006), http://dx.doi.org/10.1021/bi052629q

3. Saravanan, R., Mohanram, H., Joshi, M., Domadia, P., Torres, J., Ruedl, C., Bhattacharjya, S. Biochim. Biophys. Acta 1818, 1613-1624 (2012), http://dx.doi.org/10.1016/j.bbamem.2012.03.015

4. Wood, S.J., Park, Y.A., Kanneganti, N.P., Mukkisa, H.R., Crisman, L.L., Davis, S.E., Vandenbosch, J.L., Scaglione, J., Heyl, D.L. Int. J. Peptide Res. Ther. 20, 519-530 (2014), http://dx.doi.org/10.1007/s10989-014$\underline{9419-7}$ 